\title{
OPEN Temperature dependence of amorphous magnesium carbonate structure studied by PDF and XAFS analyses
}

\author{
Gen-ichiro Yamamoto, Atsushi Kyono ${ }^{\varpi}$ \& Satoru Okada
}

Mineral trapping through the precipitation of carbonate minerals is a potential approach to reduce $\mathrm{CO}_{2}$ accumulation in the atmosphere. The temperature dependence of amorphous magnesium carbonate (AMC), a precursor of crystalline magnesium carbonate hydrates, was investigated using synchrotron $X$-ray scattering experiments with atomic pair distribution function (PDF) and X-ray absorption fine structure analysis. PDF analysis revealed that there were no substantial structural differences among the AMC samples synthesized at 20,60 , and $80^{\circ} \mathrm{C}$. In addition, the medium-range order of all three AMC samples was very similar to that of hydromagnesite. Stirring in aqueous solution at room temperature caused the AMC sample to hydrate immediately and form a three-dimensional hydrogenbonding network. Consequently, it crystallized with the long-range structural order of nesquehonite. The Mg K-edge X-ray absorption near-edge structure spectrum of AMC prepared at $20^{\circ} \mathrm{C}$ was very similar to that of nesquehonite, implying that the electronic structure and coordination geometry of $\mathrm{Mg}$ atoms in AMC synthesized at $20^{\circ} \mathrm{C}$ are highly similar to those in nesquehonite. Therefore, the short-range order (coordination environment) around the $\mathrm{Mg}$ atoms was slightly modified with temperature, but the medium-range order of AMC remained unchanged between 20 and $80^{\circ} \mathrm{C}$.

Mineral carbonation is a promising approach for reducing the concentration of $\mathrm{CO}_{2}$ in the atmosphere ${ }^{1,2}$. There are four main trapping mechanisms that can securely store $\mathrm{CO}_{2}$, namely, structural/stratigraphic trapping, residual trapping, solubility trapping, and mineral trapping ${ }^{3-6}$. Mineral trapping involves the injection of $\mathrm{CO}_{2}$ into basalt or ultramafic rock, whereby a geochemical reaction occurs between the injected $\mathrm{CO}_{2}$ and the alkaline minerals in the basalt or ultramafic rock, leading to the precipitation of carbonate phases. This effectively locks the $\mathrm{CO}_{2}$ in immobile secondary phases for geological timescales ${ }^{6}$ and minimizes the risk of leakage to the atmosphere. The inherent stability of mineral carbonation is evidenced by the fact that more than $70 \%$ of the total carbon in the Earth's crust is present in the form of carbonates ${ }^{7}$.

The temperature, $\mathrm{CO}_{2}$ pressure, and $\mathrm{pH}$ all affect the type of crystalline magnesium carbonate hydrate that forms during carbonation. To date, 10 forms of crystalline magnesium carbonate hydrates have been recognized in the $\mathrm{MgO}-\mathrm{CO}_{2}-\mathrm{H}_{2} \mathrm{O}$ system ${ }^{8}$. Nesquehonite $\left(\mathrm{MgCO}_{3} \cdot 3 \mathrm{H}_{2} \mathrm{O}\right.$ or $\left.\mathrm{Mg}\left(\mathrm{HCO}_{3}\right)(\mathrm{OH}) \cdot 2 \mathrm{H}_{2} \mathrm{O}\right)$, hydromagnesite $\left[\mathrm{Mg}_{5}\left(\mathrm{CO}_{3}\right)_{4}(\mathrm{OH})_{2} \cdot 4 \mathrm{H}_{2} \mathrm{O}\right]$, and dypingite $\left[\mathrm{Mg}_{5}\left(\mathrm{CO}_{3}\right)_{4}(\mathrm{OH})_{2} \cdot 5 \mathrm{H}_{2} \mathrm{O}\right]$ are the most dominant phases among the crystalline magnesium carbonate hydrates. The crystal structures of nesquehonite and hydromagnesite are shown in Figs. 1 and 2, respectively. Nesquehonite (Fig. 1) is composed of infinite ribbons of $\mathrm{MgO}_{6}$ octahedra running along the $b$-axis ${ }^{8,9}$. Each $\mathrm{MgO}_{6}$ octahedra is linked to three $\mathrm{CO}_{3}$ groups lying parallel to the $c$-axis. The ribbons are highly interconnected by a hydrogen-bonding network of water molecules. In contrast, hydromagnesite (Fig. 2) has a complex structure in which sheets of $\mathrm{MgO}_{6}$ octahedra and $\mathrm{CO}_{3}$ groups are sandwiched between corrugated layers of $\mathrm{MgO}_{6}$ octahedra and $\mathrm{CO}_{3}$ groups ${ }^{10,11}$. No hydrogen-bonding network is formed in the hydromagnesite structure. The crystal structure of dypingite is still undetermined due to a lack of single-crystal specimens suitable for structural analysis, but it is thought to be similar to that of hydromagnesite ${ }^{12,13}$.

Amorphous magnesium carbonate (AMC), which can be produced by mixing aqueous solutions containing $\mathrm{Mg}^{2+}$ and $\mathrm{CO}_{3}{ }^{2-}$ ions ${ }^{14}$, is a precursor of these crystalline magnesium carbonate hydrate materials. At lower temperatures (below approximately $55^{\circ} \mathrm{C}$ ), AMC crystallizes into nesquehonite by vigorous stirring in an aqueous solution ${ }^{15,16}$, while at higher temperatures (above approximately $55^{\circ} \mathrm{C}$ ), hydromagnesite forms ${ }^{16-18}$. Although both nesquehonite and hydromagnesite grow directly from AMC at different temperatures, the structure of 
(a)
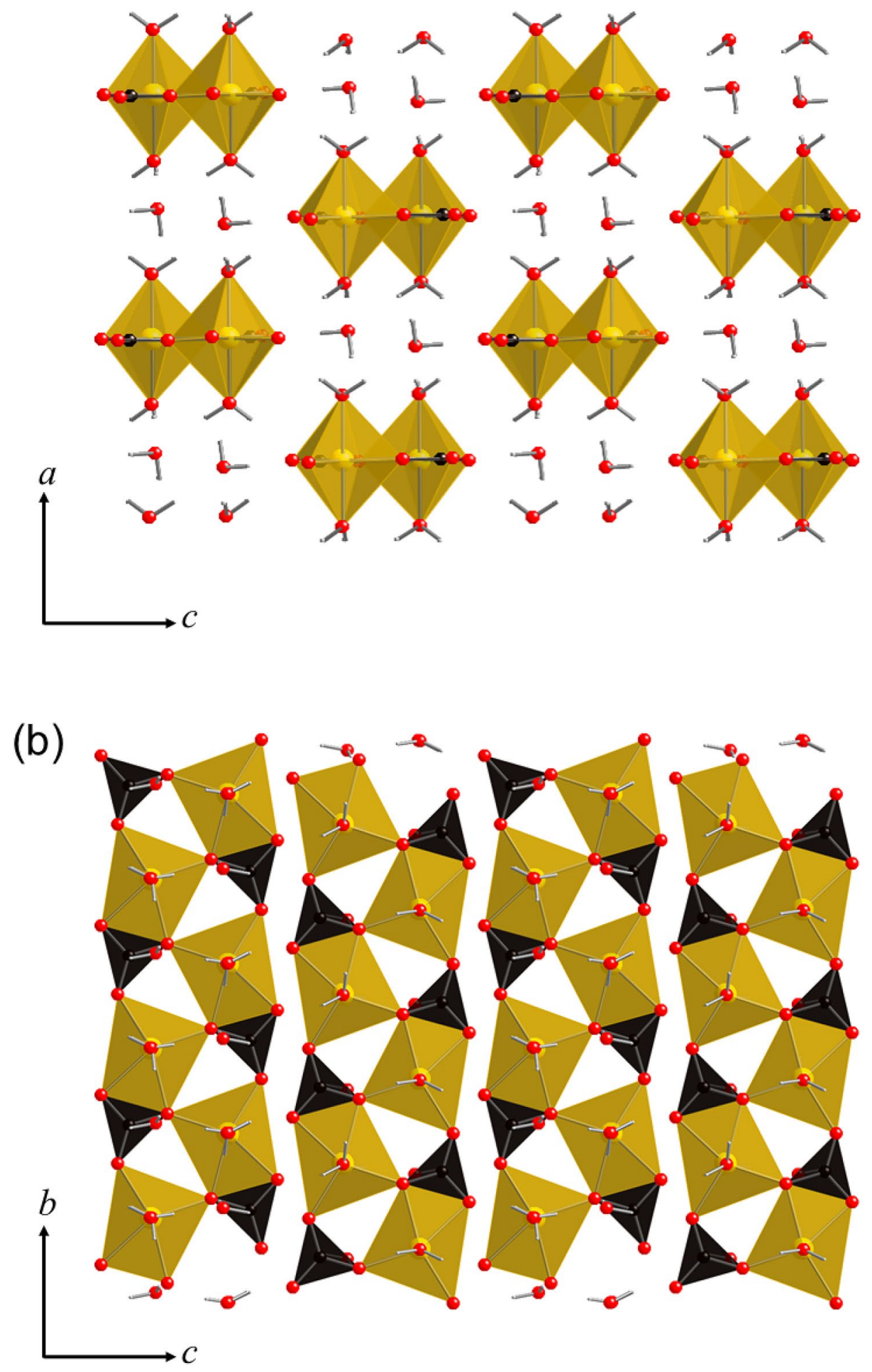

Figure 1. Crystal structure of nesquehonite $\left(\mathrm{MgCO}_{3} \cdot 3 \mathrm{H}_{2} \mathrm{O}\right)$ projected along the (a) $b$ - and (b) $a$-axes ${ }^{9}$. Yellow polyhedra and black triangles represent $\mathrm{MgO}_{6}$ octahedra and $\mathrm{CO}_{3}$ groups, respectively. Red and small white spheres denote oxygen and hydrogen atoms, respectively. The crystal structure images were generated using CrystalMaker software. 
(a)

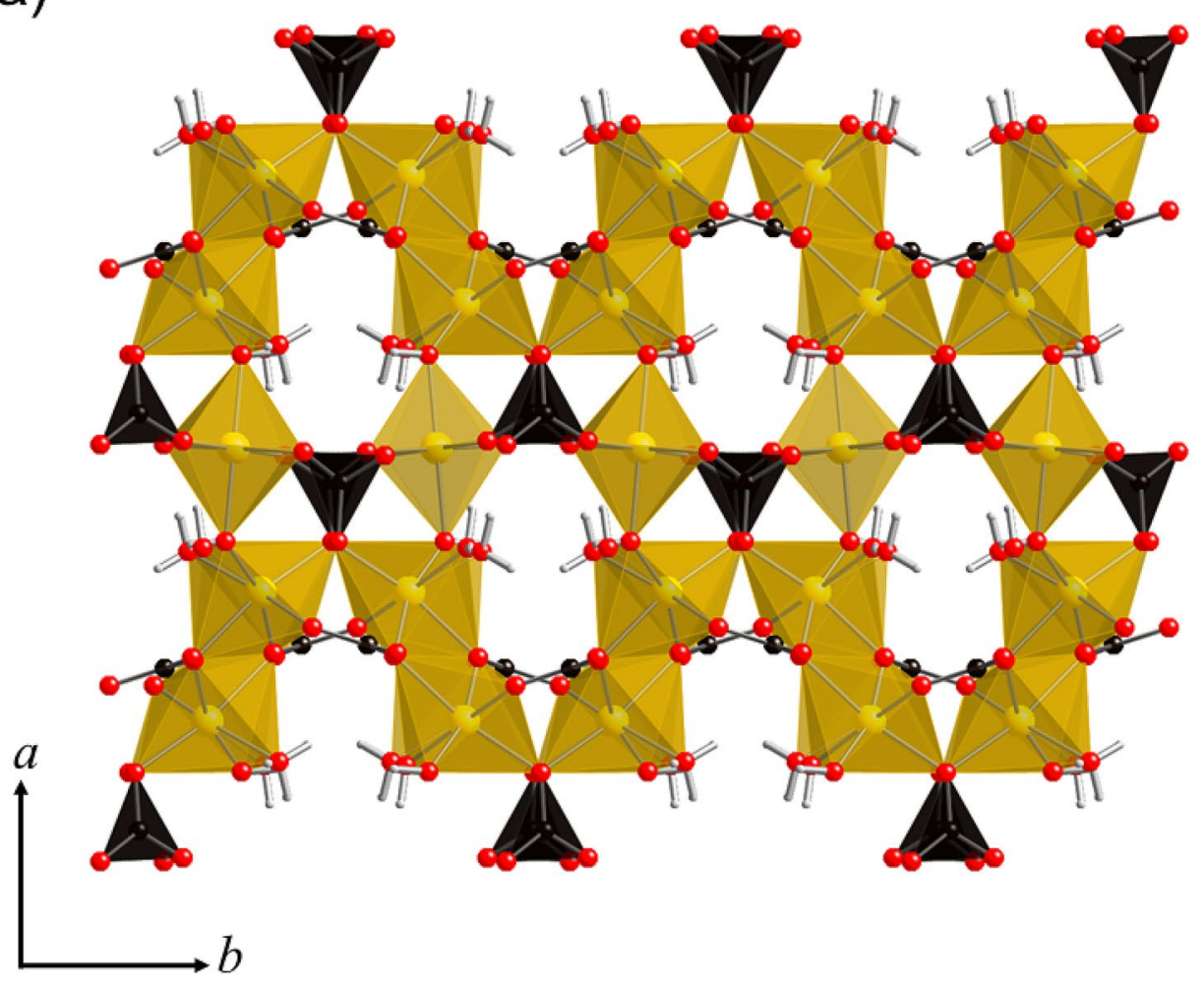

(b)

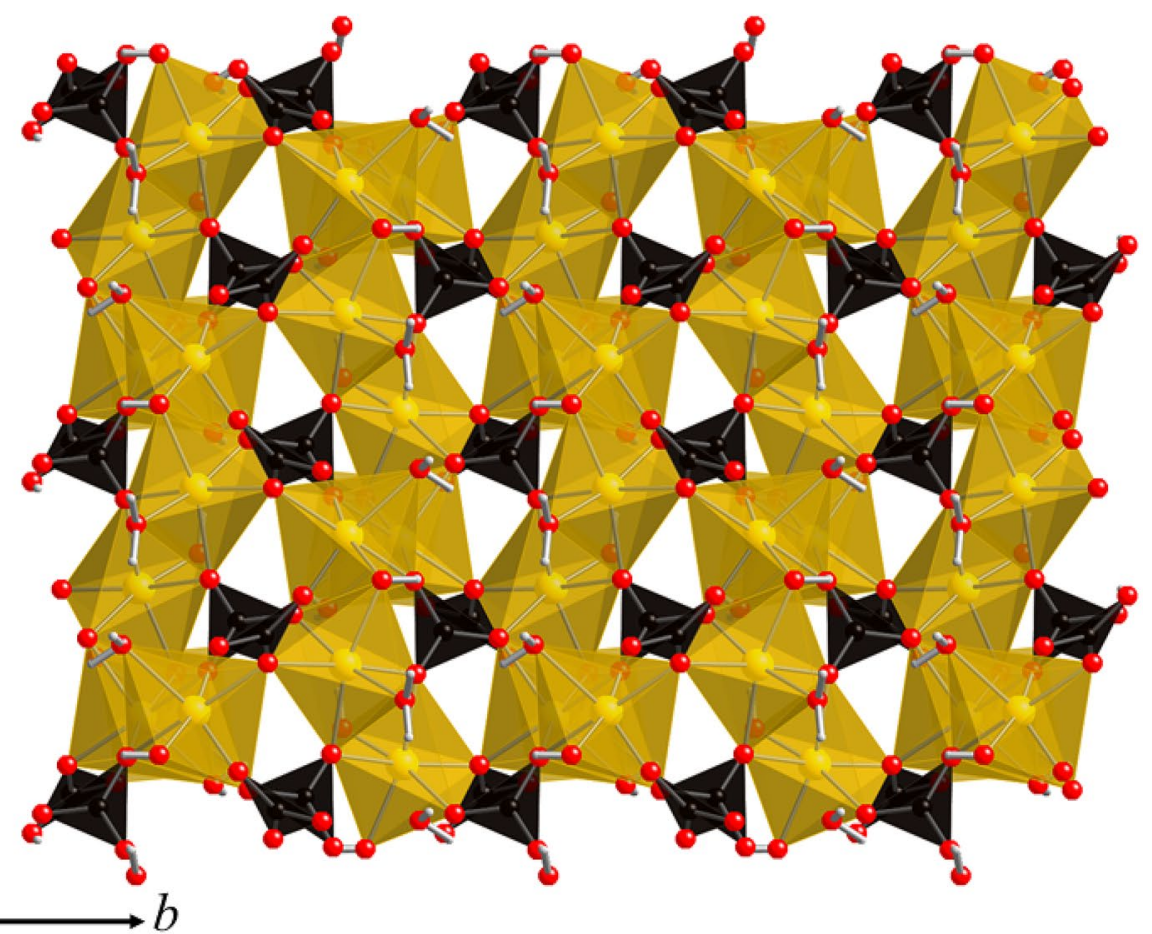

Figure 2. Crystal structure of hydromagnesite $\left[\mathrm{Mg}_{5}\left(\mathrm{CO}_{3}\right)_{4}(\mathrm{OH})_{2} \cdot 4 \mathrm{H}_{2} \mathrm{O}\right]$ projected along the (a) $c$ - and (b) $a$-axes ${ }^{10}$. Yellow polyhedra and black triangles represent $\mathrm{MgO}_{6}$ octahedra and $\mathrm{CO}_{3}$ groups, respectively. Red and small white spheres denote oxygen and hydrogen atoms, respectively. The crystal structure images were generated using CrystalMaker software. 
AMC has not been extensively investigated. In particular, it is unclear whether AMC itself has a temperaturedependent structure (e.g., a low-temperature nesquehonite-like structure and high-temperature hydromagnesitelike structure).

In this study, we investigated the temperature dependence of the AMC structure using synchrotron X-ray scattering experiments with pair distribution function (PDF) analysis and X-ray absorption fine structure (XAFS) measurements. Herein, we report the temperature dependence of the short- and medium-range structural features of AMC.

\section{Results and discussion}

AMC samples were synthesized at temperatures of 20,60 , and $80^{\circ} \mathrm{C}$ (denoted as AMC20, AMC60, and AMC80, respectively). The water content in the AMC samples was determined using thermogravimetry/differential thermal analysis (TG/DTA). The weight losses associated with dehydration were approximately 32, 29, and $26 \mathrm{wt} \%$ for AMC20, AMC60, and AMC80, respectively. Therefore, the water content of the AMC samples tended to decrease as the synthesis temperature increased. The water content corresponds to the $n$ value in the AMC chemical formula $\mathrm{MgCO}_{3} \cdot x \mathrm{H}_{2} \mathrm{O}$. From the weight loss results, it was calculated that AMC20, AMC60, and AMC80 had $x$ values of 2.2, 1.9, and 1.6, respectively, suggesting that the average chemical formula of the AMC samples is approximately $\mathrm{MgCO}_{3} \cdot 2 \mathrm{H}_{2} \mathrm{O}$, which is in good agreement with that of $\mathrm{AMC}$ obtained $15^{\circ} \mathrm{C}^{14}$. As mentioned below, since there are no substantial structural differences in AMC samples, the variation of water content does not affect the medium-range structural order of AMC.

Medium-range structural order of AMC. The X-ray PDF patterns of the AMC20, AMC60, and AMC80 samples are shown in Fig. 3a. The atom-atom correlations are considerably diminished above $5 \AA$, which indicates that the AMC samples are fully amorphous. The PDF patterns are consistent with previous reports ${ }^{19-21}$. Notably, there are no substantial structural differences in the nearest-neighbor correlations among the three AMC samples, that is, the medium-range structural order of AMC exhibits no temperature dependence in this temperature range. In other words, although the crystal structure of the crystalline magnesium carbonate hydrate produced from AMC changes with temperature (from nesquehonite to hydromagnesite via dypingite), the medium-range structural order of AMC remains unchanged. AMC acts as a pluripotent source for different magnesium carbonate hydrates.

The PDF patterns of nesquehonite, dypingite, and hydromagnesite formed by stirring the AMC samples for $2 \mathrm{~h}$ at 20,60 , and $80^{\circ} \mathrm{C}$, respectively, are shown in Fig. 3b. The PDF pattern of hydromagnesite was very similar to the PDF patterns of all three AMCs (Fig. 3a). Figure 4 shows a PDF pattern of AMC20 fitted with the hydromagnesite structure $^{10}$, demonstrating the close match between AMC20 and hydromagnesite. This indicates that the AMC samples have a similar medium-range order to that of hydromagnesite. The PDF pattern of hydromagnesite has been well characterized based on the crystal structure ${ }^{8}$. The interatomic correlation peaks at $1.3,2.1$, and $3.1 \AA$ are assigned to $\mathrm{C}-\mathrm{O}$ bonds, $\mathrm{Mg}-\mathrm{O}$ bonds, and $\mathrm{Mg}-\mathrm{Mg}$ interactions, respectively. The peak at $4.2 \AA$ corresponds to the second nearest-neighbor interaction between $\mathrm{Mg}$ and $\mathrm{O}$ atoms.

The fact that the medium-range order of AMC resembles that of hydromagnesite, even when formed at $20^{\circ} \mathrm{C}$, raises the question of why the structure of AMC20 is similar to that of hydromagnesite (grown at $80^{\circ} \mathrm{C}$ ) instead of that of nesquehonite (grown at $20^{\circ} \mathrm{C}$ ). The answer may lie in the transformation behavior of nesquehonite. AMC crystallizes into nesquehonite when stirred at room temperature (below approximately $55^{\circ} \mathrm{C}$ ), but after a few hours to a few days, it transforms to dypingite ${ }^{14,22}$. This transformation is considered to occur via a solventmediated transformation mechanism ${ }^{14}$, that is, $\mathrm{Mg}^{2+}$ and $\mathrm{CO}_{3}{ }^{2-}$ ions rapidly aggregate to form AMC with the medium-range order of hydromagnesite. By stirring the AMC in aqueous solution, it immediately hydrates and transforms to the long-range order of nesquehonite. The agitated solution environment has the advantage of forming a three-dimensional hydrogen-bonding network ${ }^{9}$. Thus, AMC with a hydromagnesite-like structure may transform into nesquehonite by hydration and crystallization processes. Considering that all the AMC samples have a hydromagnesite-like structure, the hypothesis that nesquehonite subsequently dissolves to precipitate dypingite via a solvent-mediated transformation is quite reasonable. That is, when nesquehonite dissolves in aqueous solution and returns to AMC, its structure reverts to hydromagnesite-type structure. The transformation to dypingite is therefore very advantageous because dypingite possesses a hydromagnesite-type structure as well ${ }^{12,13}$.

Short-range order of AMC. Mg K-edge X-ray absorption near-edge structure (XANES) spectroscopy is strongly dependent on the local electronegativity and multiple scattering ${ }^{23}$, which means that it can differentiate between structures with subtle modifications in local coordination and electronegativity ${ }^{24}$. This sensitivity makes it extremely useful for fingerprinting Mg-bearing phases ${ }^{24}$. The Mg K-edge XANES spectra of carbonates show prominent post-edge features between 1321 and $1350 \mathrm{eV}^{24}$. Dolomite $\left[\mathrm{MgCa}\left(\mathrm{CO}_{3}\right)_{2}\right]$ and calcite $\left(\mathrm{CaCO}_{3}\right)$ share a post-edge feature at $1324 \mathrm{eV}$, but magnesite $\left(\mathrm{MgCO}_{3}\right)$ has no such features, despite being an isostructure of dolomite and calcite, because of subtle differences in the local coordination and electronegativity.

The Mg K-edge XANES spectra of the AMC samples are shown in Fig. 5. All three spectra have similar overall shapes in the pre- and post-edge regions. Previous studies have revealed that the near-edge region between 1306 and $1321 \mathrm{eV}$ can be divided into three main components (assigned to A, B, and C) ${ }^{24-27}$. The near-edge region contributed by the three components showed a main peak centered at $1315 \mathrm{eV}$ (marked B) with shoulders at 1312 and $1320 \mathrm{eV}$ (marked A and C, respectively) (Fig. 5). A broad post-edge peak at $1330 \mathrm{eV}$ (marked E) was also observed. Notably, shoulder C was more intense for AMC60 and AMC80 than for AMC20. A similar trend was observed for the crystalline phase, that is, shoulder $\mathrm{C}$ was stronger for hydromagnesite and dypingite than for nesquehonite. Therefore, it can be considered that the short-range order (coordination geometry) around the 
(a)

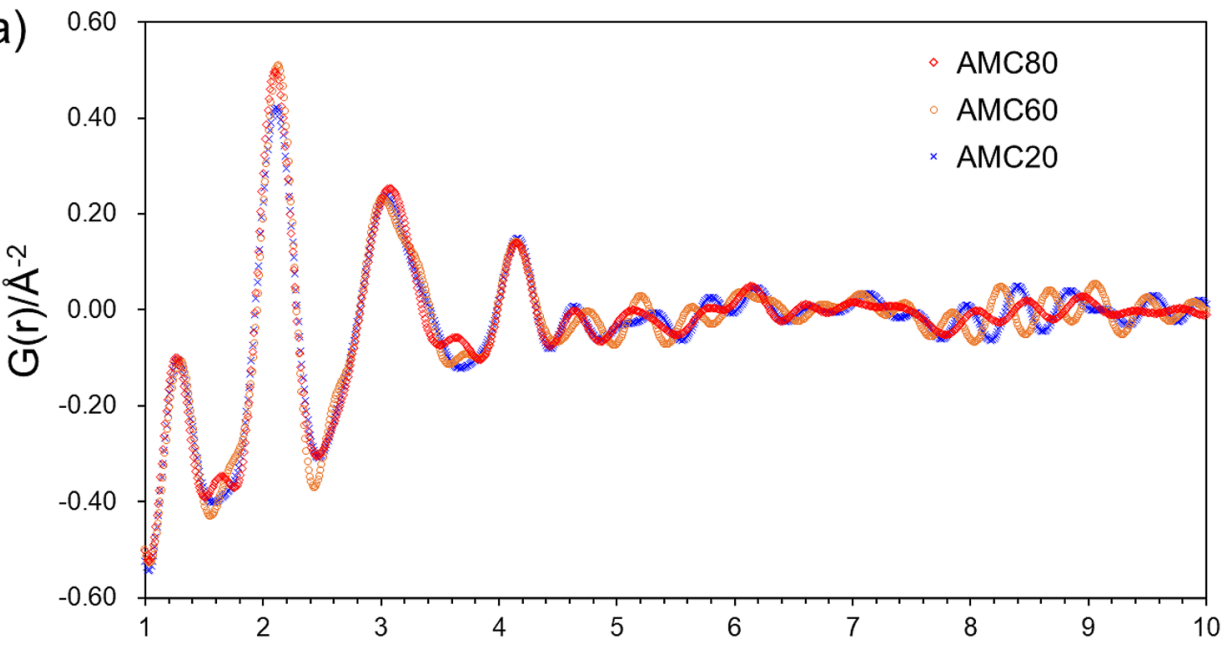

(b)

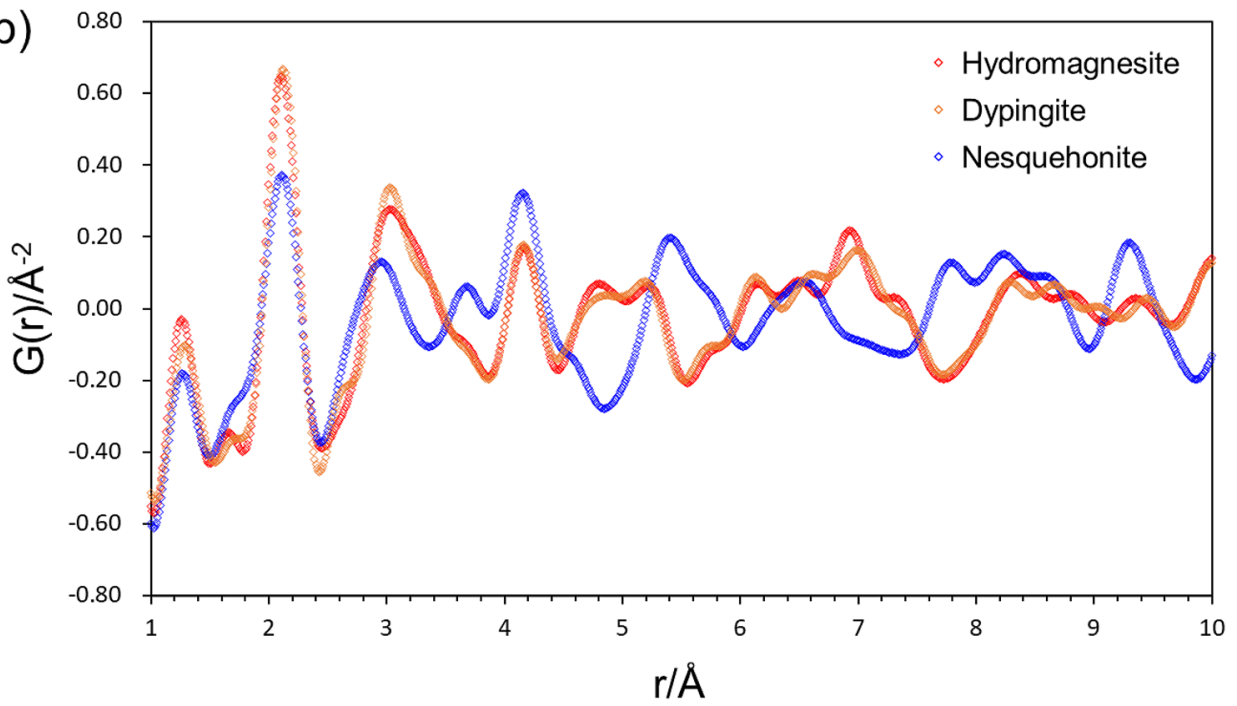

Figure 3. PDF patterns of different crystal structures. (a) AMC samples obtained at 20,60 , and $80^{\circ} \mathrm{C}$ (denoted as AMC20, AMC60, and AMC80, respectively). (b) Nesquehonite, dypingite, and hydromagnesite crystalline magnesium carbonate hydrates obtained by stirring AMC suspensions for $2 \mathrm{~h}$ at 20,60 , and $80^{\circ} \mathrm{C}$, respectively.

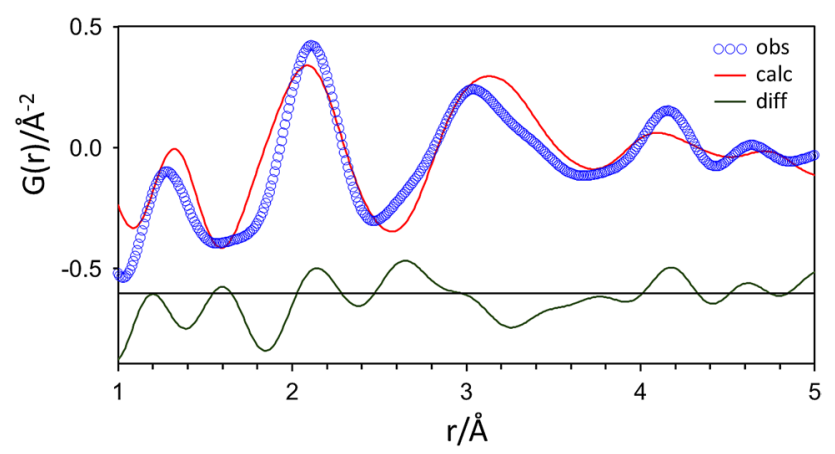

Figure 4. PDF fit of AMC20 between 1 and $5 \AA$ with hydromagnesite structure ${ }^{10}$. 


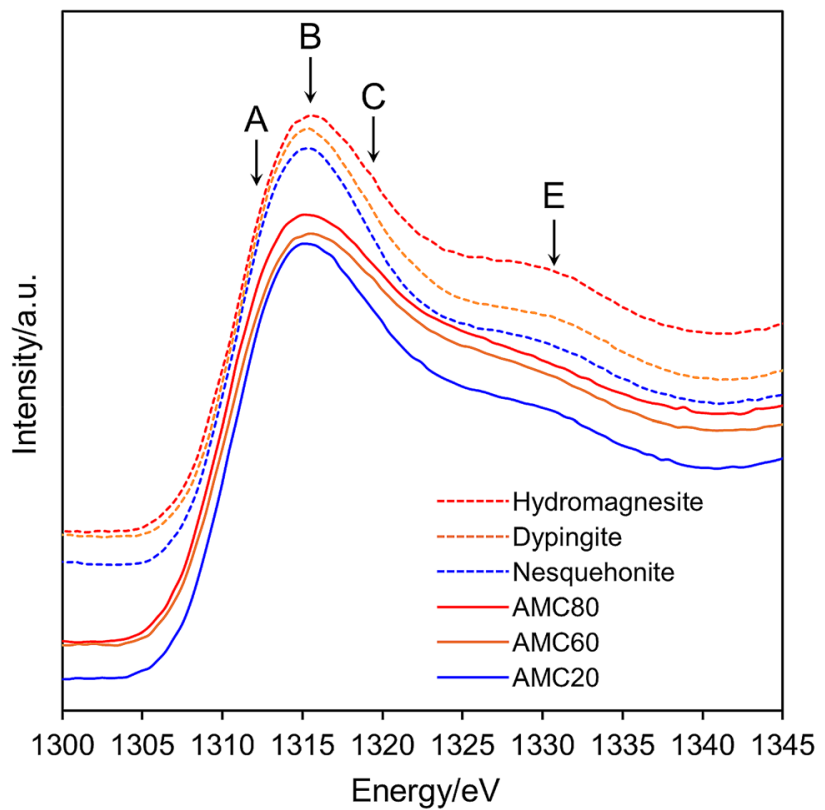

Figure 5. Mg K-edge XANES spectra of AMC samples obtained at 20, 60, and $80^{\circ} \mathrm{C}$ (denoted as AMC20, AMC60, and AMC80, respectively) and nesquehonite, dypingite, and hydromagnesite crystalline magnesium carbonate hydrates obtained by stirring AMC suspensions for $2 \mathrm{~h}$ at 20,60 , and $80^{\circ} \mathrm{C}$, respectively. The letters A to E denote each peak in the paper by Finch and Allison ${ }^{24}$.

\begin{tabular}{|c|c|c|c|c|}
\hline Mineral name & Hydromag & & & Nesquehonite \\
\hline Site & Mg1 & Mg11 & Mg2 & $\mathrm{Mg}$ \\
\hline Total energy (Hartree) & -653.087 & -653.087 & -653.082 & -653.130 \\
\hline Polyhedral volume $\left(\AA^{3}\right)$ & 11.838 & 11.953 & 11.373 & 11.408 \\
\hline Average $\mathrm{Mg}-\mathrm{O}$ bond length $(\AA)$ & 2.0858 & 2.0964 & 2.0484 & 2.0765 \\
\hline Quadratic elongation $\left\langle\lambda_{\text {oct }}\right\rangle$ & 1.0149 & 1.0189 & 1.0052 & 1.0313 \\
\hline Bond angle variance $\sigma_{\theta(\mathrm{oct})}{ }^{2}$ & 50.8 & 65.23 & 17.86 & 104.48 \\
\hline
\end{tabular}

Table 1. Total energy, polyhedral parameters, and distortion indexes of the $\mathrm{MgO}_{6}$ octahedra in hydromagnesite and nesquehonite. The distortion parameters defined by Robinson et al. ${ }^{28}$ are as follows: Quadratic elongation $\left\langle\lambda_{\text {oct }}\right\rangle=\sum_{i=1}^{6} \frac{\left(l_{i} / l_{0}\right)^{2}}{6}$, where $l_{i}$ is the bond length; $l_{0}$ is the center-to-vertex distance for an octahedron. Bond angle variance $\sigma_{\theta(\text { oct })}{ }^{2}=\sum_{i=1}^{12} \frac{\left(\theta_{i}^{i}-90^{\circ}\right)^{2}}{11}$, where $\theta_{i}$ is the $\mathrm{O}-\mathrm{Mg}-\mathrm{O}$ angle.

$\mathrm{Mg}$ atoms in AMC20 is highly similar to that in nesquehonite. In contrast, the Mg atoms in AMC60 and AMC80 have an identical coordination environment to those in hydromagnesite and dypingite.

The total energies, polyhedral parameters, and distortion indexes of the $\mathrm{MgO}_{6}$ octahedra are summarized in Table 1 . In both hydromagnesite and nesquehonite, $\mathrm{Mg}$ ions are coordinated by six oxygen atoms to form $\mathrm{MgO}_{6}$ octahedra. Assuming that all the vertices are oxygen atoms, the $\mathrm{MgO}_{6}$ octahedra in nesquehonite have a slightly lower total energy than those in hydromagnesite. In addition, the distortion parameters ${ }^{28}$ of the $\mathrm{MgO}_{6}$ octahedra in nesquehonite are larger than those in hydromagnesite. These slight differences in the electronic structure and coordination geometry of the $\mathrm{Mg}$ site contributed to the observed XANES spectra.

Consequently, the short-range order (coordination environment) around the $\mathrm{Mg}$ atoms is slightly modified depending on the synthesis temperature, but the medium-range order of AMC remains unchanged in the temperature range from 20 to $80^{\circ} \mathrm{C}$. AMC20 therefore has a hybrid structure, featuring structural characteristics of both nesquehonite and hydromagnesite. Under an agitated solution environment at room temperature, AMC crystallizes with a nesquehonite structure by forming a hydrogen-bonding network, but at elevated temperatures, it crystallizes into dypingite or hydromagnesite without any change in the short- or medium-range order.

One of the major risks related to geological $\mathrm{CO}_{2}$ sequestration is the leakage of $\mathrm{CO}_{2}$ from the storage reservoir. In this study, it was revealed that the medium-range structural order of AMC is similar to that of hydromagnesite. AMC crystallizes into nesquehonite with stirring, and then gradually transforms to dypingite, which has a hydromagnesite-like structure. Since AMC and dypingite both have hydromagnesite-like structures ${ }^{12,13}$, this suggests that the hydromagnesite-like structure is the most stable of the magnesium carbonate hydrate structures. The present and previous studies allow the prediction of the long-term stability of AMC and dypingite 
(hydromagnesite) in aqueous conditions ${ }^{14,22}$. Carbonation using AMC is likely to be the most effective sequestration technique for reducing atmospheric $\mathrm{CO}_{2}$ concentrations.

\section{Experimental procedure}

Sample preparation. Commercially available $\mathrm{MgCl}_{2}$ (Wako Pure Chemicals Co., Inc.; $\geq 97.0 \%$ purity) and $\mathrm{Na}_{2} \mathrm{CO}_{3}$ (Wako Pure Chemicals Co., Inc.; $\geq 99.5 \%$ purity) were used as the starting materials. Solutions of $0.5 \mathrm{M}$ $\mathrm{MgCl}_{2}$ and $0.5 \mathrm{M} \mathrm{Na}_{2} \mathrm{CO}_{3}$ were held at 20,60 , or $80{ }^{\circ} \mathrm{C}$, and then mixed at a $1: 1$ volume ratio. The pH values of the solutions were about 10.5 without adjustment. A white suspension of AMC formed on mixing, which was immediately filtered out and washed with distilled water heated to the same temperature, and then collected and dried overnight in air. The AMC samples obtained at 20,60, and $80^{\circ} \mathrm{C}$ were denoted as AMC20, AMC60, and AMC80, respectively. X-ray diffraction patterns for AMC20, AMC60, and AMC80 are shown in Supplementary Fig. S1. Crystalline magnesium carbonate hydrate samples were prepared by the same method, except the white suspension was stirred at about $1000 \mathrm{rpm}$ for $2 \mathrm{~h}$ after mixing. The X-ray diffraction patterns for crystalline magnesium carbonate hydrates are given in Supplementary Fig. S2. The samples prepared at 20, 60, and $80{ }^{\circ} \mathrm{C}$ with stirring for $2 \mathrm{~h}$ correspond to nesquehonite, dypingite, and hydromagnesite, respectively.

Thermal analysis. The water content in the AMC samples was determined using TG/DTA (TG/DTA-7300, Seiko Instruments Inc. Japan). Approximately $10 \mathrm{mg}$ of AMC and an $\alpha$-alumina reference were placed in $\mathrm{Al}$ pans, and subsequently heated from 50 to $550{ }^{\circ} \mathrm{C}$ at a heating rate of $10{ }^{\circ} \mathrm{C} \mathrm{min}-1$ under argon flow $\left(200 \mathrm{~mL} \mathrm{~min}^{-1}\right)$.

PDF analysis. Synchrotron X-ray total scattering measurements were performed at beamline BL22XU of SPring-8, Japan. Approximately $50 \mathrm{mg}$ of the sample was loaded into a Kapton capillary tube with an inner diameter of $2 \mathrm{~mm}$ and length of $15 \mathrm{~mm}$. The incident beam was monochromatized to a wavelength of $0.206225 \AA$ using a $\mathrm{Si}(111)$ double-crystal monochromator. Data were collected in the $Q$ range of 0.3 to $25 \AA^{-1}$. The obtained $\mathrm{X}$-ray total scattering data were transformed to total scattering structure functions $[S(Q)]$ and atomic PDFs $[G(r)]$ using PDFgetX2 software ${ }^{29}$. The obtained PDF profiles were analyzed using PDFgui software ${ }^{30}$.

XAFS analysis. Mg K-edge XAFS measurements were performed at beamline BL11A ${ }^{31}$ of the Photon Factory (PF), High Energy Accelerator Research Organization (KEK), Japan. Powder samples were mounted on carbon adhesive tape in a sample holder and placed in the experimental chamber under ultra-high vacuum. A grazing incidence monochromator served as the $\mathrm{Mg} \mathrm{K}$ absorption edge in the energy region from 1290 to $1350 \mathrm{eV}$. The samples were placed with their surface perpendicular to the incident X-ray beam. The spot size of the incident X-ray beam was approximately $2.0 \times 0.5 \mathrm{~mm}$. Energy calibration was performed using the $\mathrm{MgCO}_{3}$ spectrum.

Quantum chemical calculation. Ab initio calculations were performed using the quantum chemical calculation software package Gaussian- $09^{32}$. Nesquehonite and hydromagnesite possess $\mathrm{MgO}_{6}$ octahedra as interior structural units. Thus, the total energies of the $\mathrm{MgO}_{6}$ octahedra were calculated using the second-order Møller-Plesset (MP2) perturbation theory with the $6-311+\mathrm{G}(\mathrm{d}, \mathrm{p})$ basis set. The simulated structural model was built based on previous reports ${ }^{9,10}$. One terminal hydrogen atom was added to each oxygen atom of the $\mathrm{MgO}_{6}$ octahedra.

Received: 23 August 2021; Accepted: 12 November 2021

Published online: 24 November 2021

\section{References}

1. Oelkers, E. H., Gislason, S. R. \& Matter, J. Mineral carbonation of $\mathrm{CO}_{2}$. Elements 4, 333-337 (2008).

2. Sanna, A., Uibu, M., Caramanna, G., Kuusik, R. \& Maroto-Valer, M. M. A review of mineral carbonation technologies to sequester $\mathrm{CO}_{2}$. Chem. Soc. Rev. 43, 8049-8080 (2014).

3. McGrail, B. P. et al. Potential for carbon dioxide sequestration in flood basalts. J. Geophys. Res. 111, B12201 (2006).

4. Matter, J. M., Takahashi, T. \& Goldberg, D. Experimental evaluation of in situ $\mathrm{CO}_{2}$-water-rock reactions during CO $\mathrm{CO}_{2}$ injection in basaltic rocks: implications for geological $\mathrm{CO}_{2}$ sequestration. Geochem. Geophys. Geosyst. 8, Q02001 (2007).

5. Kelemen, P. B. et al. Rates and mechanisms of mineral carbonation in peridotite: natural processes and recipes for enhanced, in situ $\mathrm{CO}_{2}$ capture and storage. Annu. Rev. Earth Planet. Sci. 39, 545-576 (2011).

6. Aminu, M. D., Nabavi, S. A., Rochelle, C. A. \& Manovic, V. A review of developments in carbon dioxide storage. Appl. Energy 208, 1389-1419 (2017).

7. Hunt, J. M. Distribution of carbon in crust of earth. Am. Assoc. Pet. Geol. Bull. 56, 2273-2277 (1972).

8. Yamamoto, G., Kyono, A., Abe, J., Sano-Furukawa, A. \& Hattori, T. Crystal structure of nesquehonite, $\mathrm{MgCO}_{3} \cdot 3 \mathrm{H}(\mathrm{D}){ }_{2} \mathrm{O}$ by neutron diffraction and effect of $\mathrm{pH}$ on structural formulas of nesquehonite. J. Mineral. Petrol. Sci. 116, 96-103 (2021).

9. Giester, G., Lengauer, C. L. \& Rieck, B. The crystal structure of nesquehonite, $\mathrm{MgCO}_{3} \cdot 3 \mathrm{H}_{2} \mathrm{O}$, from Lavrion, Greece. Mineral. Petrol. 70, 153-163 (2000).

10. Akao, M. \& Iwai, S. The hydrogen bonding of hydromagnesite. Acta Crystallogr. B Struct. Crystallogr. Cryst. Chem. 33, 1273-1275 (1977).

11. Yamamoto, G., Kyono, A., Sano, Y., Matsushita, Y. \& Yoneda, Y. In-situ and ex-situ studies on thermal decomposition process of hydromagnesite $\mathrm{Mg}_{5}\left(\mathrm{CO}_{3}\right)_{4}(\mathrm{OH})_{2} \cdot 4 \mathrm{H}_{2} \mathrm{O}$. J. Therm. Anal. Calorim. 144, 599-609 (2021).

12. Hopkinson, L., Kristova, P., Rutt, K. \& Cressey, G. Phase transitions in the system $\mathrm{MgO}-\mathrm{CO}_{2}-\mathrm{H}_{2} \mathrm{O}$ during $\mathrm{CO}_{2}$ degassing of $\mathrm{Mg}-$ bearing solutions. Geochim. Cosmochim. Acta 76, 1-13 (2012). 
13. Yamamoto, G., Kyono, A. \& Okada, S. Thermal decomposition process of dypingite $\mathrm{Mg}_{5}\left(\mathrm{CO}_{3}\right)_{4}(\mathrm{OH})_{2} \cdot 5 \mathrm{H}_{2} \mathrm{O}$. Mater. Lett. 308, 131125 (2022).

14. Tanaka, J., Kawano, J., Nagai, T. \& Teng, H. Transformation process of amorphous magnesium carbonate in aqueous solution. J. Mineral. Petrol. Sci. 114, 105-109 (2019).

15. Kloprogge, J. T., Martens, W. N., Nothdurft, L., Duong, L. V. \& Webb, G. E. Low temperature synthesis and characterization of nesquehonite. J. Mater. Sci. Lett. 22, 825-829 (2003).

16. Morgan, B., Wilson, S. A., Madsen, I. C., Gozukara, Y. M. \& Habsuda, J. Increased thermal stability of nesquehonite $\left(\mathrm{MgCO}_{3} \cdot 3 \mathrm{H}_{2} \mathrm{O}\right)$ in the presence of humidity and $\mathrm{CO}_{2}$ : Implications for low-temperature $\mathrm{CO}_{2}$ storage. Int. J. Greenhouse Gas Control 39, 366-376 (2015).

17. Fernández, A. I., Chimenos, J. M., Segarra, M., Fernández, M. A. \& Espiell, F. Procedure to obtain hydromagnesite from a MgOcontaining residue. Kinetic study. Ind. Eng. Chem. Res. 39, 3653-3658 (2000).

18. Zhang, Z. P. et al. Temperature- and pH-dependent morphology and FT-IR analysis of magnesium carbonate hydrates. J. Phys. Chem. B 110, 12969-12973 (2006).

19. Radha, A. V. et al. Energetic and structural studies of amorphous $\mathrm{Ca}_{1-\mathrm{x}} \mathrm{Mg}_{\mathrm{x}} \mathrm{CO}_{3} \cdot \mathrm{nH}_{2} \mathrm{O}(0 \leqslant \mathrm{x} \leqslant 1)$. Geochim. Cosmochim. Acta 90, 83-95 (2012).

20. White, C. E., Henson, N. J., Daemen, L. L., Hartl, M. \& Page, K. Uncovering the true atomic structure of disordered materials: the structure of a hydrated amorphous magnesium carbonate $\left(\mathrm{MgCO}_{3} \cdot 3 \mathrm{D}_{2} \mathrm{O}\right)$. Chem. Mater. 26, 2693-2702 (2014).

21. Jensen, A. C. S., Imberti, S., Habraken, W. J. E. M. \& Bertinetti, L. Small ionic radius limits magnesium water interaction in amorphous calcium/magnesium carbonates. J. Phys. Chem. C 124, 6141-6144 (2020).

22. Montes-Hernandez, G. \& Renard, F. Time-resolved in situ Raman spectroscopy of the nucleation and growth of siderite, magnesite, and calcite and their precursors. Cryst. Growth Des. 16, 7218-7230 (2016).

23. Cabaret, D., Sainctavit, P., Ildefonse, P. \& Flank, A. M. Full multiple scattering calculations of the X-ray absorption near edge structure at the magnesium K-edge in pyroxene. Am. Mineral. 83, 300-304 (1998).

24. Finch, A. A. \& Allison, N. Coordination of Sr and Mg in calcite and aragonite. Mineral. Mag. 71, 539-552 (2007).

25. Wong, J. et al. New opportunities in XAFS investigation in the 1-2-keV region. Solid State Commun. 92, 559-562 (1994).

26. Li, D., Peng, M. \& Murata, T. Coordination and local structure of Mg in silicate minerals and glasses: Mg K-edge XANES study. Can. Mineral. 37, 199-206 (1999).

27. Trcera, N. et al. Experimental and theoretical study of the structural environment of magnesium in minerals and silicate glasses using X-ray absorption near-edge structure. Phys. Chem. Minerals 36, 241-257 (2009).

28. Robinson, K., Gibbs, G. V. \& Ribbe, P. H. Quadratic elongation: A quantitative measure of distortion in coordination polyhedra. Science 172, 567-570 (1971).

29. Qiu, X., Thompson, J. W. \& Billinge, S. J. L. PDFgetX2: A GUI-driven program to obtain the pair distribution function from X-ray powder diffraction data. J. Appl. Crystallogr. 37, 678 (2004).

30. Farrow, C. L. et al. PDFfit2 and PDFgui: Computer programs for studying nanostructure in crystals. J. Phys. Condens. Matter 19, 335219 (2007).

31. Amemiya, K., Kitajima, Y., Ohta, T. \& Ito, K. Design of a holographically recorded plane grating with a varied line spacing for a soft X-ray grazing-incidence monochromator. J. Synchrotron Radiat. 3, 282-288 (1996).

32. Frisch, M. J. et al. Gaussian 09 (Gaussian Inc, 2016).

\section{Acknowledgements}

The PDF measurements were performed at BL22XU of SPring-8 with approval of the Photon Factory Program Advisory Committee (Proposal Nos. JPMXP09A20AE0007 and JPMXP09A20AE0021). The XAFS measurements were performed at BL11A of PF KEK with approval of the Photon Factory Program Advisory Committee (Proposal No. 2021G025). This work was supported by JSPS KAKENHI (Grant Number JP20K04124).

\section{Author contributions}

All authors reviewed and approved the manuscript. G. Y.: Conceptualization, Resources, Methodology, Investigation, Data curation; A. K.: Writing, Supervision; S. O.: Investigation.

\section{Competing interests}

The authors declare no competing interests.

\section{Additional information}

Supplementary Information The online version contains supplementary material available at https:/doi.org/ 10.1038/s41598-021-02261-8.

Correspondence and requests for materials should be addressed to A.K.

Reprints and permissions information is available at www.nature.com/reprints.

Publisher's note Springer Nature remains neutral with regard to jurisdictional claims in published maps and institutional affiliations.

(c) (1) Open Access This article is licensed under a Creative Commons Attribution 4.0 International License, which permits use, sharing, adaptation, distribution and reproduction in any medium or format, as long as you give appropriate credit to the original author(s) and the source, provide a link to the Creative Commons licence, and indicate if changes were made. The images or other third party material in this article are included in the article's Creative Commons licence, unless indicated otherwise in a credit line to the material. If material is not included in the article's Creative Commons licence and your intended use is not permitted by statutory regulation or exceeds the permitted use, you will need to obtain permission directly from the copyright holder. To view a copy of this licence, visit http://creativecommons.org/licenses/by/4.0/.

(C) The Author(s) 2021 